HD-THEP-95-53

hep-ph/9512340

December 18, 1995

\title{
Perturbative Contributions to the Electroweak Interface Tension
}

\author{
Jochen Kripfganz円 \\ Andreas Laser \\ Michael G. Schmidt \\ Institut für Theoretische Physik \\ Universität Heidelberg \\ Philosophenweg 16 \\ D-69120 Heidelberg, FRG
}

\begin{abstract}
The main perturbative contribution to the free energy of an electroweak interface is due to the effective potential and the tree level kinetic term. The derivative corrections are investigated with one-loop perturbation theory. The action is treated in derivative, in heat kernel, and in a multi local expansion. The massive contributions turn out to be well described by the $Z$-factor. The massless mode, plagued by infrared problems, is numerically less important. Its perturbatively reliable part can by calculated in derivative expansion as well. A self consistent way to include the $Z$-factor in the formula for the interface tension is presented.
\end{abstract}

\footnotetext{
${ }^{1}$ supported by Deutsche Forschungsgemeinschaft

${ }^{2}$ supported by Landesgraduiertenförderung Baden-Württemberg

e-mail addresses:

J.Kripfganz@thphys.uni-heidelberg.de A.Laser@thphys.uni-heidelberg.de M.G.Schmidt@thphys.uni-heidelberg.de
} 


\section{Introduction}

The electroweak phase transition is under intense investigation [1]- [15] mostly because of possible effects in the early universe, in particular baryon number generation. Besides of this, it is the prototype of a phase transition in a gauge theory where careful understanding will be useful also in other settings, e.g. if the standard model electroweak phase transition should turn out to be too weakly first order for spectacular effects, and modified theories have to be considered.

The electroweak effective potential at high temperatures has been treated carefully in two loop order of perturbation theory [16]-[19]. Comparison with lattice nonperturbative results [4, 8, 9, 10] gives good agreement for the Higgs and $W$-boson masses in the broken phase up to zero temperature Higgs masses of about $70 \mathrm{GeV}$. The hot electroweak phase appears as a case of a confining gauge system. The Higgs and $W$-correlation masses have recently been explained by a bound state model within some approximations [15]. The critical temperature and the latent heat are expected to be influenced by nonperturbative effects in the hot (symmetric) phase. Two-loop perturbation theory agrees, nevertheless, surprisingly well with the values of these quantities measured on the lattice.

The situation for the interface tension is quite different. It is more difficult to measure on the lattice. While one lattice group finds good agreement [9] another group reports large deviations at intermediate Higgs masses [4]. Furthermore the perturbative values of the interface tension calculated from

$$
\tilde{\sigma}=\int_{\varphi_{S}}^{\varphi_{A}} d \varphi \sqrt{2 V(\varphi(r))}
$$

in the literature do not include important perturbative effects, as we will argue in this paper.

The interface tension is the free energy per area of interface between the hot and the cold electroweak phase. It is identical to the surface tension of thin walled electroweak critical bubbles which trigger the onset of the phase transition close to the critical temperature. The latter is read off from the part of the bubble free energy proportional to $4 \pi R^{2}$ ( $R=$ radius $)$.

In this paper we discuss the modification of the interface tension by one loop perturbation theory for the derivative terms of the action. In particular we address the question of whether these modification can be understood just by a $Z(\varphi)$-factor in front of the kinetic term $\partial \varphi \partial \varphi$ or whether all the other derivative terms together give a sizable effect on the interface tension.

The paper is organized as follows. In chapter 2 we recapitulate the one-loop high temperature perturbation theory. We especially address the one-loop $Z$-factor. In chapter 3 the effective action is expanded in a multi local way (section 3.1) and with the heat kernel expansion (section 3.2). The connection between both expansions and the derivative expansion is clarified in section 3.3. The infrared problems of the massless mode are investigated in chapter 4 . We show that the divergent heat kernel operators can be resummed to give a finite contribution (section 4.1). The derivative expansion of these modes is treated in detail (section 4.2). In chapter 5 a self consistent way to include the $Z$-factor in the formula for the interface tension is presented. Chapter 6 gives our conclusions.

\section{High Temperature Perturbation Theory}

The electroweak standard theory at the transition temperature allows the high temperature expansion. To good accuracy it can be described by a local effective 3-dimensional 
$S U(2)$-Higgs-model whose parameters can be calculated from the fundamental 4-dimensional theory [3]. All infrared problems appearing in the unbroken phase are covered by this effective theory. The nature of the phase transition depends substantially on the static Matsubara frequencies. Calculating the one-loop effective action of a scalar background field in the derivative expansion using a general 't Hooft background gauge with gaugefixing parameter $\xi$ one gets 20, 19]

$$
S_{\mathrm{eff}}[\varphi]=\frac{1}{g_{3}(T)^{2}} \int d^{3} r\left[V_{\mathrm{eff}}(\varphi)+\frac{1}{2} Z_{\mathrm{H}}(\varphi) \partial_{i} \varphi \partial_{i} \varphi+\mathcal{O}\left(\partial^{4}\right)\right]
$$

with the effective potential

$$
\begin{aligned}
V_{\mathrm{eff}}(\varphi) & =V_{\mathrm{ht}}(\varphi)-\frac{g_{3}(T)^{2}}{12 \pi}\left(9 m_{W}^{3}-6 m_{\mathrm{gh}}^{3}+3 m_{\mathrm{Gs}}^{3}+3 m_{\mathrm{H}}^{3}\right) \\
V_{\mathrm{ht}}(\varphi) & =\frac{\lambda_{T}}{g^{2}}\left(\frac{\varphi^{4}}{4}-\left(\frac{v_{0}(T)}{v}\right)^{2} \frac{\varphi^{2}}{2}\right)
\end{aligned}
$$

and the $Z$-factor

$$
\begin{aligned}
Z_{\mathrm{H}}(\varphi)=1+\frac{g_{3}^{2}}{4 \pi} & \left\{-\frac{3}{m_{\mathrm{Gs}}+m_{W}}+\frac{1}{m_{W}^{2}}\left(\frac{m_{\mathrm{Gs}}^{3}-m_{W}^{3}}{m_{\mathrm{Gs}}^{2}-m_{W}^{2}}-\frac{m_{\mathrm{Gs}}^{3}-\xi^{3 / 2} m_{W}^{3}}{m_{\mathrm{Gs}}^{2}-\xi m_{W}^{2}}\right)\right. \\
& +\frac{10-13 \sqrt{\xi}+9 \xi}{16(1+\sqrt{\xi})} \frac{1}{m_{W}^{3}}\left(\frac{\partial m_{W}^{2}}{\partial \varphi}\right)^{2}-\frac{2}{16} \frac{1}{m_{\mathrm{gh}}^{3}}\left(\frac{\partial m_{\mathrm{gh}}^{2}}{\partial \varphi}\right)^{2} \\
& \left.+\frac{1}{16} \frac{1}{m_{\mathrm{Gs}}^{3}}\left(\frac{\partial m_{\mathrm{Gs}}^{2}}{\partial \varphi}\right)^{2}+\frac{1}{48} \frac{1}{m_{\mathrm{H}}^{3}}\left(\frac{\partial m_{\mathrm{H}}^{2}}{\partial \varphi}\right)^{2}\right\} .
\end{aligned}
$$

The squared high temperature Higgs, Goldstone, $W$-boson and ghost masses are

$$
\begin{aligned}
m_{\mathrm{H}}^{2} & =\frac{\lambda_{T}}{g^{2}}\left(3 \varphi^{2}-\left(\frac{v_{0}(T)}{v}\right)^{2}\right), & m_{W}^{2} & =\frac{1}{4} \varphi^{2} \\
m_{\mathrm{Gs}}^{2} & =\frac{\lambda_{T}}{g^{2}}\left(\varphi^{2}-\left(\frac{v_{0}(T)}{v}\right)^{2}\right)+\frac{1}{4} \xi \varphi^{2}, & m_{\mathrm{gh}}^{2} & =\frac{1}{4} \xi \varphi^{2} .
\end{aligned}
$$

$\lambda_{T}$ and $v_{0}(T)$ can be calculated from the fundamental 4-dimensional parameters. We identify

$$
\frac{\lambda_{T}}{g^{2}}=\frac{\bar{m}_{\mathrm{H}}^{2}}{8 m_{W}(T=0)^{2}}
$$

The mass $\bar{m}_{\mathrm{H}}$ is of order of the zero temperature Higgs mass. $v_{0}(T)$ is the temperature dependent "high temperature tree level" vacuum expectation value of the complex scalar field (cf. eq. (国)). $v$ was used to rescale fields and will be chosen to be the field value at the broken minimum later on. $g_{3}(T)^{2}=g T / v$ is the rescaled dimensionless coupling constant of the 3 -dimensional theory. In this respect we differ from the notation $g_{3}^{2}=g^{2} T$ sometimes being used.

The two-loop effective potential has been calculated by different methods [16]-[18]. We use the general 't Hooft background gauge potential obtained in [19].

The different contributions to $Z_{\mathrm{H}}(\varphi)$ (eq. (5)) are due to different graphs. The first two terms in the brackets originate from a mixed $W$-Gs-loop. The other corrections are generated by a pure $W$-boson, ghost, Goldstone, or Higgs loop respectively. The use of the derivative expansion is limited for several reasons:

First of all $Z_{\mathrm{H}}(\varphi)$ diverges as $\varphi$ goes to 0, i.e. in the symmetric phase, due to the massless $W$-boson. At this order of derivative and loop expansion the effective action (2) 
is nevertheless finite, since a bubble solution vanishes exponentially in this phase. For dimensional reasons the next order of the derivative expansion behaves like $m_{W}{ }^{-5}(\partial \varphi)^{4}$ for small $\varphi$. The two-loop order is expected to give a $m_{W}{ }^{-2}(\partial \varphi)^{2}$ and a $m_{W}{ }^{-2} \ln \left(m_{W}\right)(\partial \varphi)^{2}$ term. All these terms lead to a diverging action. Thus perturbation theory breaks down. Note that even the effective potential diverges at four-loop order for $\varphi \rightarrow 0$. The divergences are cured by a finite $W$-boson mass in the symmetric phase due to confining forces.

Second, $Z_{\mathrm{H}}(\varphi)$ becomes negative in some $\varphi$-range depending on $\xi$ (e.g. for $\bar{m}_{\mathrm{H}} \gtrsim 45 \mathrm{GeV}$ at $\xi=1$ ). This is due to the numerical dominance of the mixed $W$-Gs-loop (cf. section 3.1). Here again perturbation theory in connection with the derivative expansion is in bad shape, since the one loop contribution is larger than the tree part. In addition a negative $Z$-factor gives rather unphysical results and no critical bubbles at all.

Finally $Z_{\mathrm{H}}(\varphi)$ depends strongly on the gauge-fixing parameter even in the broken phase, as we have shown in ref. [19]. Therefore higher derivative terms and/or the two-loop order are expected to give important contributions to $Z_{\mathrm{H}}(\varphi)$. The gauge-fixing dependence of the potential diminishes from one to two-loop, and it may be hoped that the same happens with the $Z$-factor.

\section{Higher Order Derivative Terms}

It is an obvious question how important the $\mathcal{O}\left(\partial^{4}\right)$-terms in eq. (2) are. Calculating the next order in the derivative expansion would not by a feasible way to investigate this question because of the divergence in the symmetric phase due to massless modes. One has to use different methods to expand the effective action. In ref. [20] we treated the Higgs fluctuation separately using the heat kernel method but we did not calculate the contribution of the $W$-boson and the Goldstone fluctuation to the interface tension. In this paper we will include these contribution. We use two different methods of expansion: the heat kernel method and a multi local expansion. To the required order for both methods the 't Hooft-Feynman background gauge (i.e. $\xi=1$ ) is more convenient. We therefore restrict ourselves in this chapter to this gauge-fixing.

The one-loop effective action including all derivative corrections is

$$
S_{\mathrm{eff}}[\varphi]=S_{\mathrm{ht}}[\varphi]+\delta S[\varphi]
$$

with the high temperature tree level part

$$
S_{\mathrm{ht}}=\frac{1}{g_{3}(T)^{2}} \int d^{3} r\left[\frac{1}{2} \partial_{i} \varphi \partial_{i} \varphi+V_{\mathrm{ht}}\left(\varphi^{2}\right)\right]
$$

and the one-loop correction

$$
\delta S=\frac{1}{2} \ln \operatorname{det}\left(-\partial^{2}+U_{13}\right)-\ln \operatorname{det}\left(-\partial^{2}+U_{\text {gh }}\right) .
$$

where $U_{13}$ is the $13 \times 13$-matrix

$$
U_{13}=\left(\begin{array}{cccc}
U_{4} & 0 & 0 & 0 \\
0 & U_{4} & 0 & 0 \\
0 & 0 & U_{4} & 0 \\
0 & 0 & 0 & m_{\mathrm{H}}^{2}
\end{array}\right) \quad U_{4}=\left(\begin{array}{cccc}
m_{W}^{2} & 0 & 0 & \partial_{1} \varphi \\
0 & m_{W}^{2} & 0 & \partial_{2} \varphi \\
0 & 0 & m_{W}^{2} & \partial_{3} \varphi \\
\partial_{1} \varphi & \partial_{2} \varphi & \partial_{3} \varphi & m_{\mathrm{Gs}}^{2}
\end{array}\right)
$$

and the $3 \times 3$-ghost matrix is $m_{\text {gh }}^{2} 1_{3 \times 3}$. The diagonal elements are the finite temperature squared Higgs, Goldstone, $W$-boson and ghost masses (eq. (6)). 
We proceed now in choosing a typical interface profile which depends of course on $\lambda_{T}$ (i.e. on the zero temperature Higgs and top mass). Then the determinants in eq. (10) are evaluated numerically. The contributions of $\delta S$ to the effective potential are removed. The remaining one-loop correction is compared with the contribution of the $Z$-factor.

A typical interface between the symmetric phase $(\varphi=0)$ and the broken phase $(\varphi=1)$ is given by the thin wall configuration:

$$
\varphi(z)=\frac{1}{2}\left(1-\tanh \left(\frac{z-z_{0}}{d}\right)\right)
$$

The wall thickness $d$ depends on the quartic coupling $\sqrt{\lambda_{T} / g^{2}}$. At the critical temperature its tree $+\varphi^{3}$-level value in units of $(g v)^{-1}$ is given by

$$
d=\frac{\sqrt{8}}{\sqrt{\lambda_{T} / g^{2}}}=\frac{8 \sqrt{\pi}}{\sqrt{g_{3}^{2}}} .
$$

For small $\lambda_{T} / g^{2}$ an expansion in $d^{-1}$ seems to be appropriate. $d$ may be considered as a variational parameter.

The interface eq. (12) does not depend on the $x$ - or $y$-coordinate of $\vec{r}$. The matrix $U_{13}$ of eq. (11) simplifies and is partly canceled by the ghost matrix. The one-loop effective action (eq. (10)) becomes

$$
\delta S=\frac{3}{2} \ln \operatorname{det}\left(-\partial^{2}+U_{2}\right)+\frac{1}{2} \ln \operatorname{det}\left(-\partial^{2}+m_{\mathrm{H}}^{2}\right) .
$$

with the $2 \times 2$-matrix

$$
U_{2}=\left(\begin{array}{cc}
m_{W}^{2} & \partial_{z} \varphi \\
\partial_{z} \varphi & m_{\mathrm{Gs}}^{2}
\end{array}\right)
$$

In order to evaluate the one-loop action (eq. (14)) approximately one may choose several different directions to go.

A) The derivative expansion discussed above. There is the obvious problem of infrared divergences blowing up the integrated action if one expands beyond the $Z$-factor.

B) The Barvinsky-Vilkovisky expansion [21] where one fixes the number of fields and sums over all derivatives, just opposite to the derivative expansion, obtaining form factors.

C) The heat kernel expansion where derivatives and fields are expanded according to their mass dimension and hence mixed together. This approach is in between (A) and (B).

An accessible way to treat (B) will be given in the next section 3.1. This multi local expansion will automatically sum all derivative terms for a given power of fields. After this, we will study the heat kernel expansion in section 3.2 .

\subsection{The Multi Local Expansion}

The one-loop contributions to the effective action have the general structure (cf. eq. (14)):

$$
\Gamma=\frac{1}{2} \ln \operatorname{det} K=\frac{1}{2} \operatorname{Tr} \ln K
$$

where the matrix $K$ is $\left(-\partial^{2}+U(\vec{r})\right) \cdot U(\vec{r})$ is either $U_{2}$ or the $1 \times 1$-matrix $\left(m_{\mathrm{H}}^{2}\right)$ in our case but may be different in other settings. We split $K$ into two parts

$$
K=K_{0}+U_{I}(\vec{r}), \quad K_{0}=\left(-\partial^{2}+m_{0}^{2}\right), \quad U_{I}(\vec{r})=U(\vec{r})-m_{0}^{2}
$$


$K_{0}$ is $\vec{r}$-independent while the interaction part $U_{I}(\vec{r})$ depends on the background field. $m_{0}$ is arbitrary and not a physical mass. But it should be of the order of the typical mass scale of the problem to improve the convergence of the series we are generating.

One gets

$$
\begin{aligned}
\Gamma & =\frac{1}{2} \operatorname{Tr} \ln K_{0}\left(1+K_{0}{ }^{-1} U_{I}\right) \\
& =\frac{1}{2} \operatorname{Tr} \ln K_{0}+\frac{1}{2} \operatorname{Tr} \ln \left(1+K_{0}{ }^{-1} U_{I}\right) \\
& =\frac{1}{2} \operatorname{Tr} \ln K_{0}-\frac{1}{2} \operatorname{Tr} \sum_{n=1}^{\infty} \frac{(-1)^{n}}{n}\left(K_{0}{ }^{-1} U_{I}\right)^{n} .
\end{aligned}
$$

The first term of the right hand side of eq. (20) does not give any contribution to the interface tension. The second term can be evaluated in coordinate space

$$
\frac{1}{2} \sum_{n=1}^{\infty} \frac{(-1)^{n}}{n} \int d r_{1} \ldots \int d r_{n} U_{I}\left(\vec{r}_{1}\right) P\left(\vec{r}_{1}, \vec{r}_{2}, m_{0}\right) \ldots U_{I}\left(\vec{r}_{n}\right) P\left(\vec{r}_{n}, \vec{r}_{1}, m_{0}\right)
$$

The main advantage of the splitting of $K$ is that the 'propagator' $K_{0}{ }^{-1}$ can be calculated exactly

$$
P\left(\vec{r}_{i}, \vec{r}_{j}, m_{0}\right)=\frac{1}{4 \pi\left|\vec{r}_{i}-\vec{r}_{j}\right|} \exp \left(-m_{0}\left|\vec{r}_{i}-\vec{r}_{j}\right|\right) .
$$

This is not possible for an arbitrary $\vec{r}$-dependent mass.

Equation (21) contains all corrections to the kinetic terms included in $\Gamma$ (eq. (16)). It also gives contributions to the effective potential which can be removed if one uses the connection of the derivative expansion to the multi local expansion (cf. section 3.3). The remaining part, which we are calculating, is independent of $m_{0}$ if summed to all orders, while the volume part gets a $m_{0}$-dependent contribution from the first term of the right hand side of eq. (20). Truncating the sum in eq. (21) at finite order $N$ results of course in a $m_{0}$-dependent interface contribution denoted by $\Gamma_{N}\left(m_{0}\right)$. Looking for the $m_{0}$-independent range gives a good criterion for the convergence of the series.

The multi local expansion can be adjusted to the interface (eq. (12)) which depends only on one of three spatial coordinates. Fourier transforming $P\left(\vec{r}_{i}, \vec{r}_{j}, m_{0}\right)$ of eq. (22) with respect to $x$ and $y$ one gets

$$
\tilde{P}\left(z_{i}, z_{j}, m_{0}, q\right)=\frac{1}{2 \sqrt{m_{0}^{2}+q^{2}}} \exp \left(-\sqrt{m_{0}^{2}+q^{2}}\left|z_{i}-z_{j}\right|\right), \quad q^{2}=p_{x}^{2}+p_{y}^{2}
$$

The $q$-integral in each term of eq. (21) can now be performed analytically. The remaining integrals over the $z_{i}$ 's have to be calculated numerically. They are convergent for $m_{0}>0$.

Similar to the derivative expansion one can distinguish between different contributions to the interface tension. There are those which originate from single Higgs, Goldstone, $W$, or ghost-loops, and those which correspond to mixed $W$-Goldstone loops. The ghost-loop cancels $2 / 3$ of the $W$-loop contribution. The $W$-loops do not have an intrinsic mass scale since $m_{W} \rightarrow 0$ in the symmetric phase. The others are massive all over the interface.

Fig. 11 shows typical results of our calculations for $\lambda_{T} / g^{2}=0.024$ and $d=18.3$ which corresponds to a zero temperature Higgs mass of about $35 \mathrm{GeV}$. Fig. 1.a shows $\Gamma_{N}\left(m_{0}\right)$ $(N=2 \ldots 7)$ for a typical massive loop (the Higgs mode) while fig. 1.b shows the same quantity for the $W$-mode. It turns out that the series behaves badly for small $m_{0}$. If $m_{0}$ is similar to the mass in the middle of the interface or larger one gets good convergence for the Higgs, the Goldstone, and the mixed $W$-Goldstone loop. The $W$ and ghost loop contributions even in this range converge very slowly. 

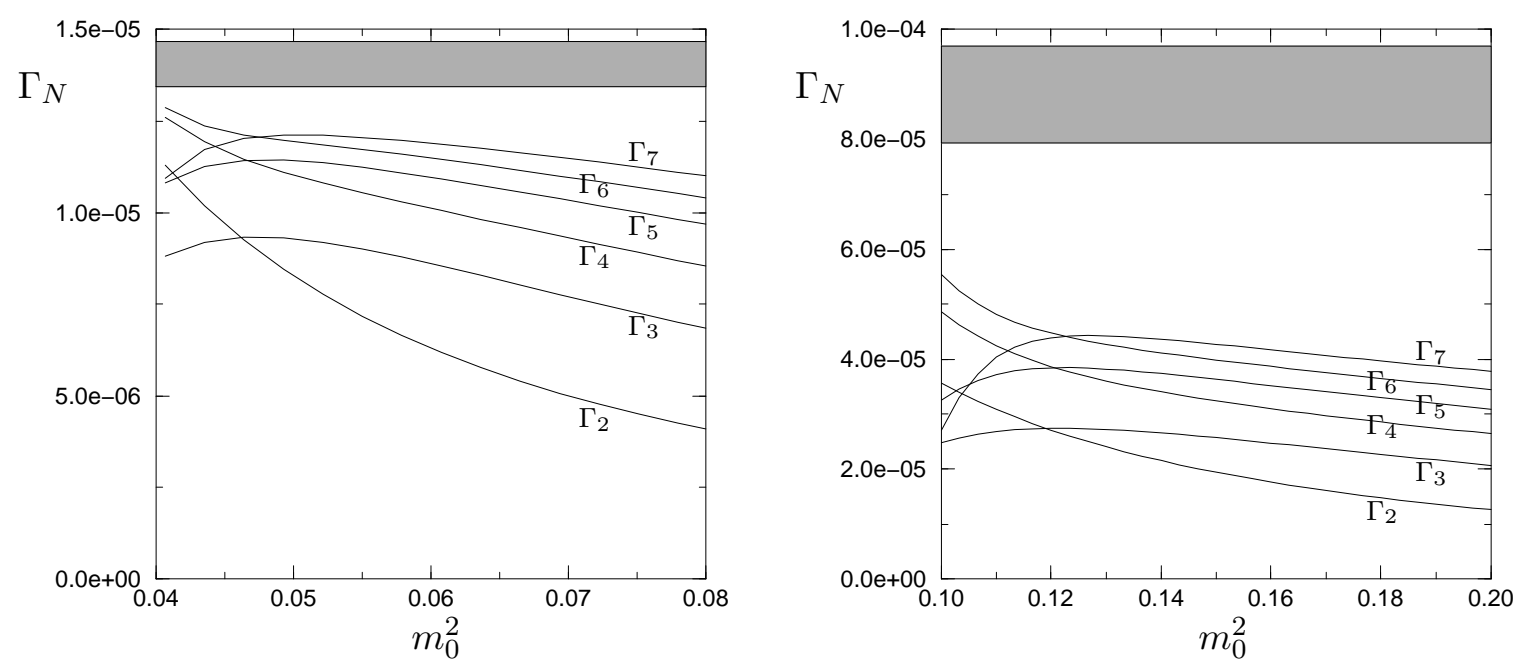

Figure 1: $\Gamma_{N}\left(m_{0}\right)$ vs. $m_{0}^{2}$. Left plot: the Higgs-fluctuation, right plot: the $W$-fluctuation. The limits of extrapolation are expected to be in the hatched range. The parameters are: $\lambda_{T} / g^{2}=0.024, d=18.3$ corresponding to $\bar{m}_{\mathrm{H}}=35 \mathrm{GeV}$.

We extrapolated $\Gamma_{N}\left(m_{0}\right)$ to $N=\infty$ for several values of $m_{0}$. From the $m_{0}$-dependence of the results we estimated an error of the limit. In fig. 2 the limits with error bars of our extrapolations are plotted for several values of $\bar{m}_{\mathrm{H}}$ (eq. (7)). Fig. 2. a shows the single Higgs, Goldstone and $W$-contributions (single modes) while fig. 2 .b shows the mixed $W$-Gsloop. The full lines are the predictions from the $Z$-factor for the corresponding quantities.

One finds small deviations for the single modes (less than 10\%). The $W$-Gs-contribution agrees within the errors with the $Z$-factor prediction. The large errors are due to the extrapolation to $N=\infty$. At any finite order the deviations are smaller as we will show in section 3.3. The $W$-Gs-contribution is an order of magnitude larger than the sum of the single modes contributions. This depends of course on the gauge-fixing parameter $\xi$ which is 1 in our case.

\subsection{The Heat Kernel Expansion}

Another way to calculate the higher derivative terms of the one-loop effective action is the heat kernel expansion [22]. $\Gamma$ (eq. (16)) is expressed as

$$
\Gamma=\frac{1}{2} \int_{0}^{\infty} \frac{d t}{t} \exp \left(-m_{0}^{2} t\right) \operatorname{Tr} \exp \left(-t\left(-\partial^{2}+U_{I}\right)\right)
$$

$m_{0}$ and $U_{I}(\vec{r})$ are defined in the same way as above. $\operatorname{Tr} \exp \left(-t\left(-\partial^{2}+U_{I}\right)\right)$ is now expanded in powers of $t$. Using world-line methods this can be done in a very economical way. One finally gets

$$
\Gamma=\frac{1}{2} \int_{0}^{\infty} \frac{d t}{t}(4 \pi t)^{-d / 2} \exp \left(-m_{0}^{2} t\right) \sum_{n=1}^{\infty}(-t)^{n} O_{n}
$$

where the $O_{n}$ 's are functionals of the field $\varphi(\vec{r})$. In reference [23] they are given up to $\mathrm{O}_{8}$; with the new world-line method they can easily be calculated to even higher order. For $n>d / 2$ the $t$-integral gives $\Gamma(n-d / 2)$. The dimension $d$ is in our case 3 . Using 

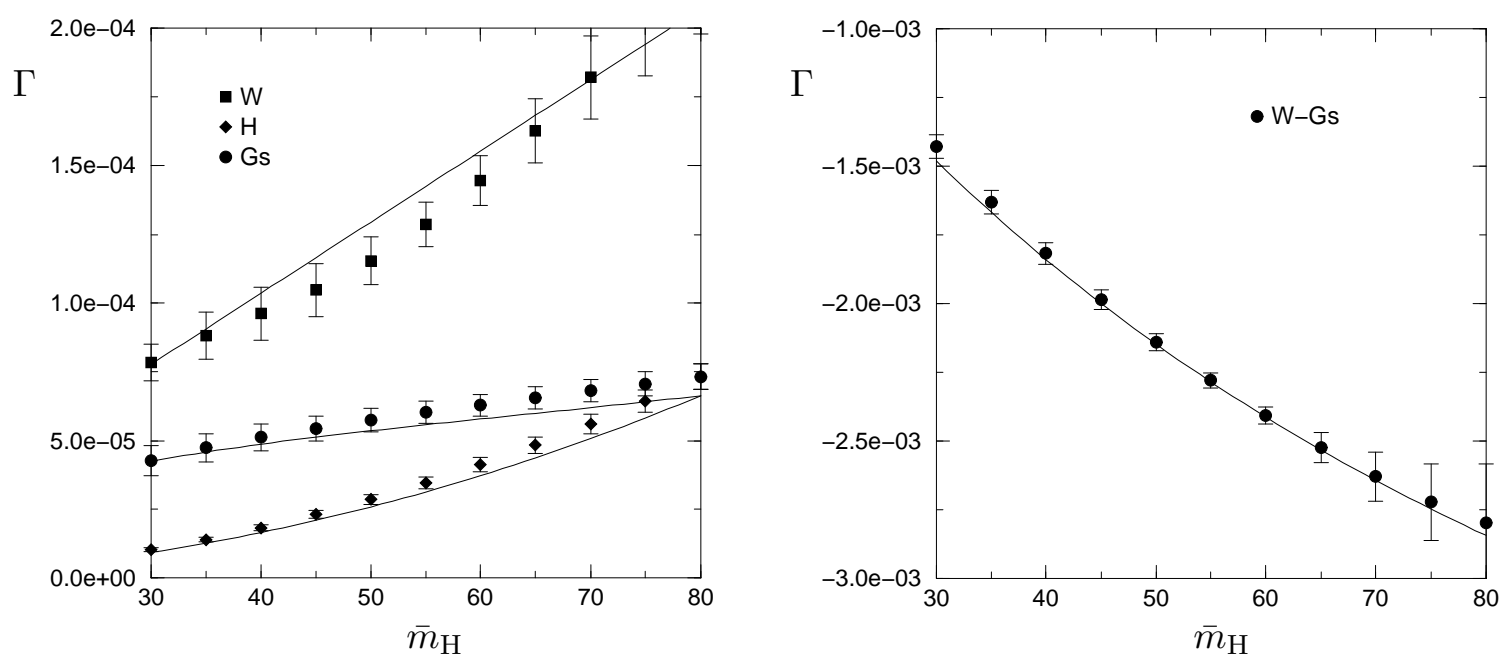

Figure 2: Extrapolated results of the multi local expansion and the $Z$-factor predictions vs. $\bar{m}_{\mathrm{H}}$ (eq. 甲). The full lines are the $Z$-factor predictions.

this formula for $n \leq d / 2$ as well defines a dimensional regularization of the ultraviolet divergences.

Every order of this expansion is again free of infrared divergences for $m_{0}>0$. Truncating the sum at finite order $N$ one generates $m_{0}$-dependent terms although the limit is independent of $m_{0}$. This aspect is very similar to the multi local expansion. Indeed even the numerical results are nearly the same if one compares the $N$ th order of the multi local expansion with the $(N+1)$ th order of the heat kernel expansion, though the higher derivatives are truncated in the heat kernel approach. One can again extrapolate to infinite order. The results agree very well with those of the multi local expansion. This shows that at low order of the corresponding expansion higher order contributions in $1 / d$ do not play any significant role at small $\lambda_{T}$ (large $d$ ). Possible large expansion parameters do not arise. This need not be the case at higher orders, as discussed in chapter 1 .

\subsection{Connection between Derivative, Multi Local, and Heat Kernel Ex- pansion}

The good agreement between the limit of large $N$ of the multi local expansion and of the heat kernel expansion confirms that our results are reliable. The agreement is already very good at small $N$ and this indicates that the higher derivative terms are numerically very small. Indeed the large- $N$ limit is very well approximated by the $Z$-factor prediction. In order to separate the higher derivative terms in the two expansions it is necessary to know how the effective potential and the $Z$-factor build up.

The operators $O_{n}$ of the heat kernel expansion are already local. One can sort directly for powers of derivatives. The terms without explicit derivatives are of the form

$$
\frac{1}{2} \int_{0}^{\infty} \frac{d t}{t}(4 \pi t)^{-d / 2} \exp \left(-m_{0}^{2} t\right) \sum_{n=1}^{\infty}(-t)^{n} \frac{1}{n !} \int d^{3} r U_{I}(\vec{r})^{n}
$$

If one has a $1 \times 1$-matrix $U_{I}=\left(m(\vec{r})^{2}-m_{0}^{2}\right)$ these terms sum up to give the $m(\vec{r})^{3}$-term of the effective potential (cf. eq. (3)) . 


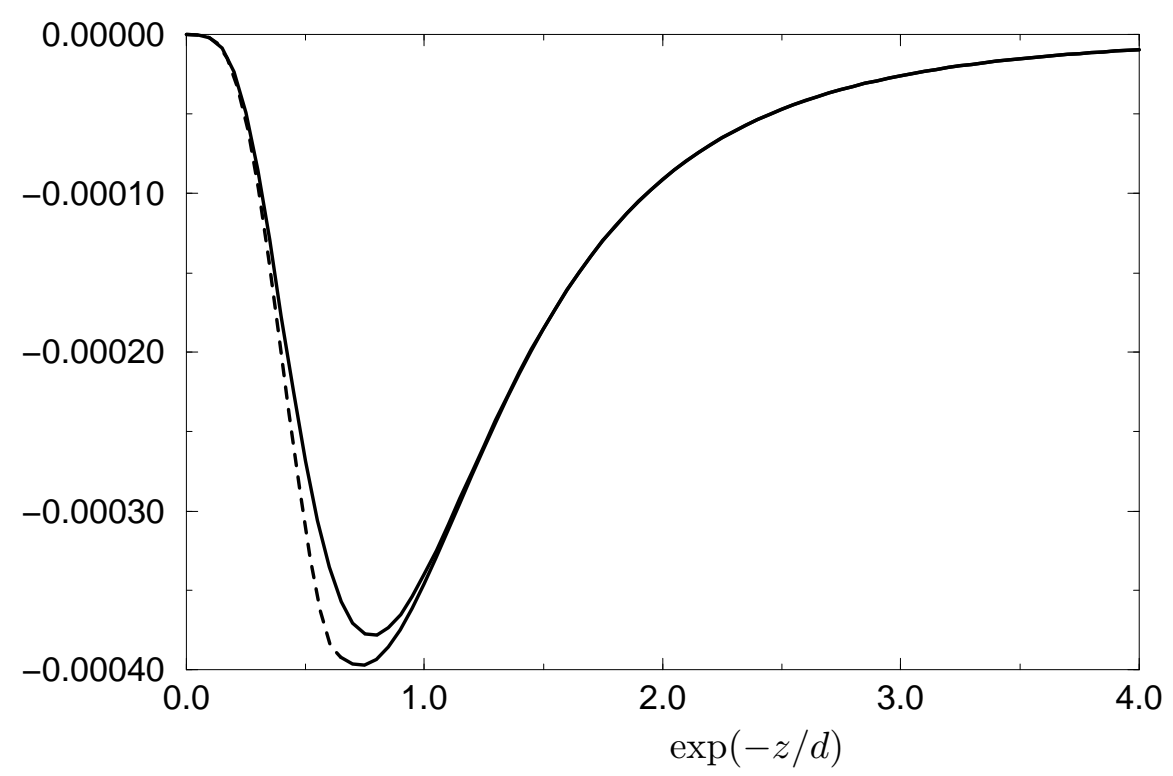

Figure 3: The integrand of eq. (29) and the $Z$-factor prediction vs. $\exp (-z / d)$. The parameters are $\lambda_{T} / g^{2}=0.04, d=12.5$

If $U_{I}$ is the $2 \times 2$-matrix $\left(U_{2}-m_{0}^{2}\right)$, with $U_{2}$ of eq. (15), even this term contains derivatives. The two-derivative order of this term gives the mixed $W$-Gs-loop contribution to $Z_{\mathrm{H}}$ (eq. (5)). $U_{2}$ can be diagonalized to give

$$
\left(\begin{array}{ccc}
\frac{1}{2} m_{W}^{2}+\frac{1}{2} m_{\mathrm{Gs}}^{2}+\frac{1}{2} \sqrt{m_{W}^{4}-2 m_{W}^{2} m_{\mathrm{Gs}}^{2}+m_{\mathrm{Gs}}^{4}+4\left(\partial_{z} \varphi\right)^{2}} & 0 \\
0 & \frac{1}{2} m_{W}^{2}+\frac{1}{2} m_{\mathrm{Gs}}^{2}-\frac{1}{2} \sqrt{m_{W}^{4}-2 m_{W}^{2} m_{\mathrm{Gs}}^{2}+m_{\mathrm{Gs}}^{4}+4\left(\partial_{z} \varphi\right)^{2}}
\end{array}\right)
$$

In the background of the interface (eq. (12)) one eigenvalue $\lambda_{+}$is positive over the whole $\varphi$-range while the second one $\lambda_{-}$turns negative for small $\varphi$. Summing up all orders of $n$ one gets

$$
\int d^{3} r\left(\lambda_{+}(\vec{r})^{3 / 2}+\lambda_{-}(\vec{r})^{3 / 2}\right)
$$

The contribution to the surface tension is proportional to the $z$-integral of

$$
-3\left(\lambda_{+}^{3 / 2}+\lambda_{-}^{3 / 2}-m_{\mathrm{Gs}}^{3}-m_{W}^{3}\right)
$$

In fig. 3 the integrand is plotted in comparison to the $Z$-factor prediction for this contribution. In the region where $\lambda_{-}$is negative the real part has been taken. This is shown by the dashed line. One sees that the $Z$-factor prediction is a very good approximation over the whole $z$ (respectively $\varphi$ ) range.

The terms in the heat kernel expansion which have two explicit derivatives are of the form

$$
\frac{1}{24} \int_{0}^{\infty} \frac{d t}{t}(4 \pi t)^{-d / 2} \exp \left(-m_{0}^{2} t\right)(-t)^{3} \int d^{3} r(\partial U)^{2} \frac{1}{n !}\left(-U_{I} t\right)^{n}
$$

For a single mode they sum up to the $Z$-factor contributions. Nevertheless, this series converges only slowly in the massless channel. Since the higher derivative terms are numerically small this is the main reason for the bad convergence of the heat kernel expansion for the massless mode. The same applies to the multi local expansion (cf. fig. 1. 1.b).

If one is interested only in the higher derivative terms one has to subtract those terms which contribute to the $Z$-factor. The remaining contribution to the interface tension 
turns out to be very small $(\lesssim 1 \%$ ) compared to the $Z$-factor contribution to all orders we calculated. This again suggests that it can be neglected in good accuracy.

The connection between the multi local and the derivative expansion is established by expanding every order locally. If one substitutes in eq. (21) the integration variables via

$$
\begin{aligned}
\vec{R} & =\frac{1}{n}\left(\vec{r}_{1}+\ldots+\vec{r}_{n}\right) \\
\vec{\rho}_{1} & =\vec{r}_{2}-\vec{r}_{1}, \ldots, \quad \vec{\rho}_{n-1}=\vec{r}_{n}-\vec{r}_{n-1}
\end{aligned}
$$

and Taylor-expands $U_{I}\left(\vec{r}_{i}\right)$ around $\vec{R}$ the $\vec{\rho}_{i}$-integrals can be done analytically. Summing up all terms without derivatives one gets the effective potential. We used this connection to separate the interface from the volume contributions.

Summing up all terms with two derivatives regenerates the $Z$-factor contribution. The multi local expansion is an expansion in powers of fields. Therefore this contribution builds up by terms which contain two derivatives and $2 N$ fields. Comparing with eq. (30) one confirms that these must be the same terms which connect the heat kernel expansion with the derivative expansion. Hence the $Z$-factor contribution is generated in both expansions in the same way. The $N$ th order of the multi local expansion corresponds to the $(N+1)$ th order of the heat kernel expansion. If the higher derivative terms are small one expects the corresponding orders of both expansions to give similar results. This behavior was indeed observed by us.

In conclusion of this chapter both methods, the multi local and the heat kernel expansion, although quite different from the calculational point of view, yield the same results because the higher derivative terms are very small at the order of the expansion that has been studied. For interfaces typical for zero temperature Higgs masses up to $80 \mathrm{GeV}$ the $Z$-factor of the derivative expansion gives numerically nearly all corrections to the kinetic term of the action. The massless mode ( $W$-boson) would need higher order treatment in these expansions. The additional contribution is expected to be numerically unimportant. In the next chapter we investigate this mode in more detail.

\section{The Massless Mode}

\subsection{Resummation of Infrared Divergent Heat Kernel Operators}

For "small" Higgs mass $m_{\mathrm{H}} \leq m_{W}$, i.e. small $\lambda_{T} / g^{2}$, and $g_{3}^{2}$ the wall thickness $d$ (in units of $\left.\left(g v_{0}(T)\right)^{-1}\right)$ is large (cf. eq. (13)). This suggests a large- $d$ expansion of the one-loop contributions to $\sigma(d)$. The appropriate tool would to be the derivative expansion

$$
\sigma(d)=c_{\partial^{0}} d+\frac{c_{\partial^{2}}}{d}+\frac{c_{\partial^{4}}}{d^{3}}+\mathcal{O}\left(\frac{1}{d^{5}}\right)
$$

where the first term corresponds to the potential, the next one to the wave function contribution, and the following to the four derivative terms of the effective action. This expansion, however, breaks down due to infrared singularities coming from the massless degrees of freedom as we have shown above. Already the $c_{\partial^{4}}$-term does not exist.

Obviously the question comes up whether one can rearrange the various contributions in such a way that a meaningful large- $d$ expansion arises. It will turn out that the first two terms in eq. (33) are not modified and remain leading. The next term (in the massless sector) will be of order $d^{-2}$ not present in the derivative expansion. In order to see this behavior and to isolate this contribution it is useful to sum up "towers" of related terms in the heat kernel expansion "vertically". This means that one does not evaluate the complete heat kernel coefficient but only parts. These parts have the same number of derivatives 
and are labeled with $\alpha$. If one does the summation without caring about divergences one regenerates the derivative expansion but this is not correct in the infrared region. Here it is better to use the exponential falloff of the background to rearrange the series.

Setting $m_{0}=0$ in eq. (30) one sees that the one-loop wave function contribution to the interface tension is given by a tower of terms of the form $t^{n} U(z)^{n}(\partial U(z))^{2}$. These are generated by an exponential $\exp (-U t)(\partial U)^{2}$

$$
\sigma_{\partial^{2}}=\frac{c_{\partial^{2}}}{d}=\frac{1}{24} \int_{0}^{\infty} \frac{d t}{t}(4 \pi t)^{-3 / 2} t^{3} \int d z(\partial U)^{2} \exp (-U t)
$$

At higher orders of $t^{n}$ we obtain the general structure

$$
\sigma_{\alpha, \beta}=\frac{1}{2} \int_{0}^{\infty} \frac{d t}{t}(4 \pi t)^{-3 / 2} t^{\beta+\alpha / 2} \int d z O_{\alpha \beta} \exp (-U t)
$$

where the first index $(\alpha \geq 2)$ is the number of derivatives and the second one $(0 \leq \beta \leq \alpha)$ labels the towers summed over; $\beta$ is equal to the power of $U$ 's. A similar rearrangement of the heat kernel operators was already obtained in ref. [24]. Up to order six the operators $O_{\alpha, \beta}$ are given by

$$
\begin{aligned}
O_{2,2} & =\frac{1}{12}(\partial U)^{2} \\
O_{4,2} & =-\frac{1}{120}(\partial \partial U)^{2} \\
O_{4,3} & =\frac{1}{72} \partial \partial U(\partial U)^{2} \\
O_{4,4} & =-\frac{105}{30240}(\partial U)^{4} \\
O_{6,2} & =\frac{1}{1680}(\partial \partial \partial U)^{2} \\
O_{6,3} & =-\frac{1}{30240}\left(22(\partial \partial U)^{3}+84 \partial \partial \partial U \partial \partial U \partial U\right) \\
O_{6,4} & =\frac{1}{9979200}\left(30030(\partial \partial U)^{2}(\partial U)^{2}+7700(\partial \partial \partial U)(\partial U)^{3}\right) \\
O_{6,5} & =-\frac{300300}{259459200}\left((\partial \partial U)(\partial U)^{4}\right) \\
O_{6,6} & =\frac{1051050}{10897286400}(\partial U)^{6}
\end{aligned}
$$

Using eq. (35) for the massless $W$ and ghost modes the $t$-integrals are infrared divergent, i.e. for $t \rightarrow \infty$, because of the behavior of $U(z)=m_{W}(z)^{2}=\varphi(z)^{2} / 4$ at $z \rightarrow \infty$. With the ansatz eq. (12) $U$ behaves as

$$
\begin{aligned}
U(z \rightarrow \infty) & =\frac{1}{4} \exp (-4 z / d) \\
\partial U(z \rightarrow \infty) & =-\frac{4}{d} U(z) .
\end{aligned}
$$

Using this interface shape we can now introduce $U$ as integration variable

$$
\frac{d z}{d}=-\frac{1}{4} \frac{d U}{U}
$$

and rewrite eq. (35) as

$$
\begin{aligned}
\sigma_{\alpha, \beta} & =c_{\alpha, \beta} \int_{0}^{\infty} \frac{d t}{t}(4 \pi t)^{-3 / 2} t^{\beta+\alpha / 2}\left(\frac{-4}{d}\right)^{\alpha-1} \int_{0}^{\epsilon} \frac{d U}{U} U^{\beta} \exp (-U t) \\
& =c_{\alpha, \beta} \int_{0}^{\infty} \frac{d t}{t}(4 \pi t)^{-3 / 2}\left(\frac{t}{d^{2}}\right)^{\alpha / 2} d(-4)^{\alpha-1} f(\beta) .
\end{aligned}
$$


$f(\beta)$ is the $U$-integral rescaled with $t$.

$$
f(\beta)=\int_{0}^{\epsilon t} \frac{d u}{u} u^{-\beta} \exp (-u) \underset{\substack{d \rightarrow \infty \\ t / d^{2} \text { fixed }}}{\longrightarrow} \Gamma(\beta)
$$

The upper integration limit $\epsilon$ must be small enough for the approximation eq. (36) to be valid. The remaining part of the $z$-integration in eq. (35) is not related to the infrared part and needs no resummation. The two derivative term $\alpha=2$ is finite and can be treated separately as well. It generates the one-loop contributions to the $Z$-factor.

The $\alpha \geq 3$ terms in eq. (40) diverge at large $t$. All these terms have to be resummed. Rescaling $\tilde{t}=t / d^{2}$ we get

$$
\sigma_{\alpha, \beta} \cong c_{\alpha, \beta}(-4)^{\alpha-1} \Gamma(\beta) \frac{1}{d^{2}} \int_{0}^{\infty} \frac{d \tilde{t}}{\tilde{t}}(4 \pi \tilde{t})^{-3 / 2} \tilde{t}^{\alpha / 2}
$$

The $c_{\alpha, \beta}$ 's are

\begin{tabular}{|rr|r|r|}
\hline$\alpha$ & $\beta$ & $c_{\alpha, \beta} \Gamma(\beta)$ & $c_{\alpha}=\sum_{\beta} c_{\alpha, \beta} \Gamma(\beta)$ \\
\hline 4 & 2 & -2.133 & \\
& 3 & 7.111 & -0.356 \\
& 4 & -5.333 & \\
\hline 6 & 2 & 2.438 & \\
& 3 & -28.715 & \\
& 4 & 92.919 & \\
& 5 & -113.718 & 0.271 \\
& 6 & 47.497 & \\
\hline
\end{tabular}

This clearly shows strong cancelations between towers of operators starting in different order of the heat kernel expansion. This should have a deeper reason which we still do not understand.

The contribution from the higher derivative terms is summarized by

$$
\sum_{\alpha \geq 4, \beta} \sigma_{\alpha, \beta} \cong \frac{1}{d^{2}} \int_{0}^{\infty} \frac{d \tilde{t}}{\tilde{t}} \frac{1}{(4 \pi \tilde{t})^{3 / 2}} \sum_{\alpha \geq 4}(-4)^{\alpha-1} c_{\alpha} \tilde{t}^{\alpha / 2}
$$

This equation is of course only a formal expression since the $\tilde{t}$-integrals do not exist in the infrared $(\tilde{t} \rightarrow \infty)$ term by term. It is likely, however, that the complete 1-loop contribution has a $1 / d$ expansion, perhaps in the sense of an asymptotic series. This would correspond to a resummation of the series $\sum_{\alpha \geq 4}(-4)^{\alpha-1} c_{\alpha} \tilde{t}^{\alpha / 2}$ in such a way that the $\tilde{t}$-integral exists. We did not attempt to prove this assertion because the heat kernel expansion is not an appropriate tool for this purpose. Determining $c_{\alpha}$ involves heat kernel contributions up to order $3 \alpha / 2$. We saw dramatic cancelations between the different heat kernel contributions of different order to the same $c_{\alpha}$. A more appropriate framework for calculating the coefficient of the $1 / d^{2}$ term might be the treatment of the eigenvalue spectrum of the equivalent quantum mechanical problem with the Hamiltonian $-\partial_{z}^{2}+\frac{1}{4} \varphi(z)^{2}$.

The $1 / d^{2}$ contribution arises from the region $t \sim d^{2}$ and can not be seen in the multi local or the heat kernel expansion in finite order because these expansions cover only a finite $t$ range (in units of $v$ ). The natural order of magnitude for this non-leading correction to the predicted $Z$-factor contribution of the massless mode is therefore $5-10 \%$ at intermediate Higgs mass (assuming a coefficient of order one). 


\subsection{Derivative Expansion with a Non-Perturbative Infrared Cutoff}

Because the infrared region is sensitive to non-perturbative effects (confinement) one could also take the point of view of cutting-off this contribution by introducing a magnetic mass $m_{\gamma}=\gamma g^{2} T$. Strictly speaking non-perturbative effects also change the vertex functions. Introducing a cut-off mass is therefore at best an approximate tool. It would enter in eq. (42) as a factor $\exp \left(-m_{\gamma}^{2} d^{2} \tilde{t}\right)$. The exponent can easily be estimated using eq. (13) and $g_{3}(T)=g T / v(T)$

$$
m_{T}^{2} d^{2}=\gamma^{2} g^{4} T^{2} \frac{8}{\lambda_{T} / g^{2}}(g v(T))^{-2}=\gamma^{2}\left(\frac{g T}{v(t)}\right)^{2} \frac{8}{\lambda_{T} / g^{2}}=\gamma^{2} 64 \pi g_{3}^{2} .
$$

This is not small for any interesting Higgs mass, if $\gamma \gtrsim 1 /(3 \pi)$ (cf. ref. [25]). Therefore possible large $\tilde{t}$, i.e. infrared, contributions to eq. (42) are strongly suppressed if one introduces such a cut off mass $m_{\gamma}$. If such contributions would become big in perturbation theory this would be a typical infrared effect and not be physical, because perturbation theory is not applicable in this range. However, we do not expect that.

Since the higher derivative terms turn out to be small and therefore the effective action apparently is well described by the potential and the $\partial \varphi \partial \varphi$ term it might be useful to look to the derivative expansion again.

There are three scales in the problem.

(i) The inverse wall thickness

$$
\frac{1}{d}=\sqrt{\frac{\lambda_{T}}{8 g^{2}}} g v(T)
$$

derivatives of the interface have this dimension.

(ii) The $\vec{r}$-dependent field

$$
g \frac{\Phi(\vec{r})}{\sqrt{2}}=g v(T) \varphi(\vec{r}) .
$$

( $\varphi(\vec{r})$ is normalized to 1 in the broken phase)

(iii) A genuine tree mass $m_{3}$ in the Higgs and Goldstone mode and an infrared scale $m_{\gamma}$ in the massless modes, respectively. We will denote both masses with $m$ in this section.

The derivative expansion is valid if

$$
\text { a) } \frac{1}{d}<m \quad \text { or } \quad \text { b) } \quad \frac{1}{d}<g \frac{\Phi(\vec{r})}{\sqrt{2}}
$$

since a typical behavior of a tower with $\alpha$ derivatives starting at power $\beta$ of $\Phi^{2}$ is

$$
\left(\frac{1}{d}\right)^{\alpha} /\left(\frac{g^{2} \Phi(\vec{r})^{2}}{4}+m^{2}\right)^{\alpha / 2+\beta-3 / 2} .
$$

a) means $d^{2} m_{3}^{2}>1$ or at the critical temperature in the massless channel $d^{2} m_{\gamma}^{2}=$ $\gamma^{2} g_{3}^{2} 64 \pi>1$ (eq. (44)). Given some (unknown) value of $\gamma(\gamma=\mathcal{O}(1) /(3 \pi)$ in the work of ref. 25]) this is not fulfilled for correspondingly small $g_{3}(T)^{2}$ (i.e. $\lambda_{T}$ small).

b) means

$$
\varphi>\frac{\sqrt{\lambda_{T} / g^{2}}}{\sqrt{8}} \sim \frac{m_{\mathrm{H}}}{8 m_{W}} \quad \text { at tree level. }
$$

Thus for large $m_{\mathrm{H}}>m_{W}$, i.e. $g_{3}^{2}$ on the upper end limit of perturbation theory, small magnetic masses can be specified because $(a)$ is fulfilled. For small $m_{\mathrm{H}}$ the range of allowed $\varphi$ 's extends almost to $\varphi=0$ with condition $(b)$, though condition $(a)$ might not be fulfilled anymore. 


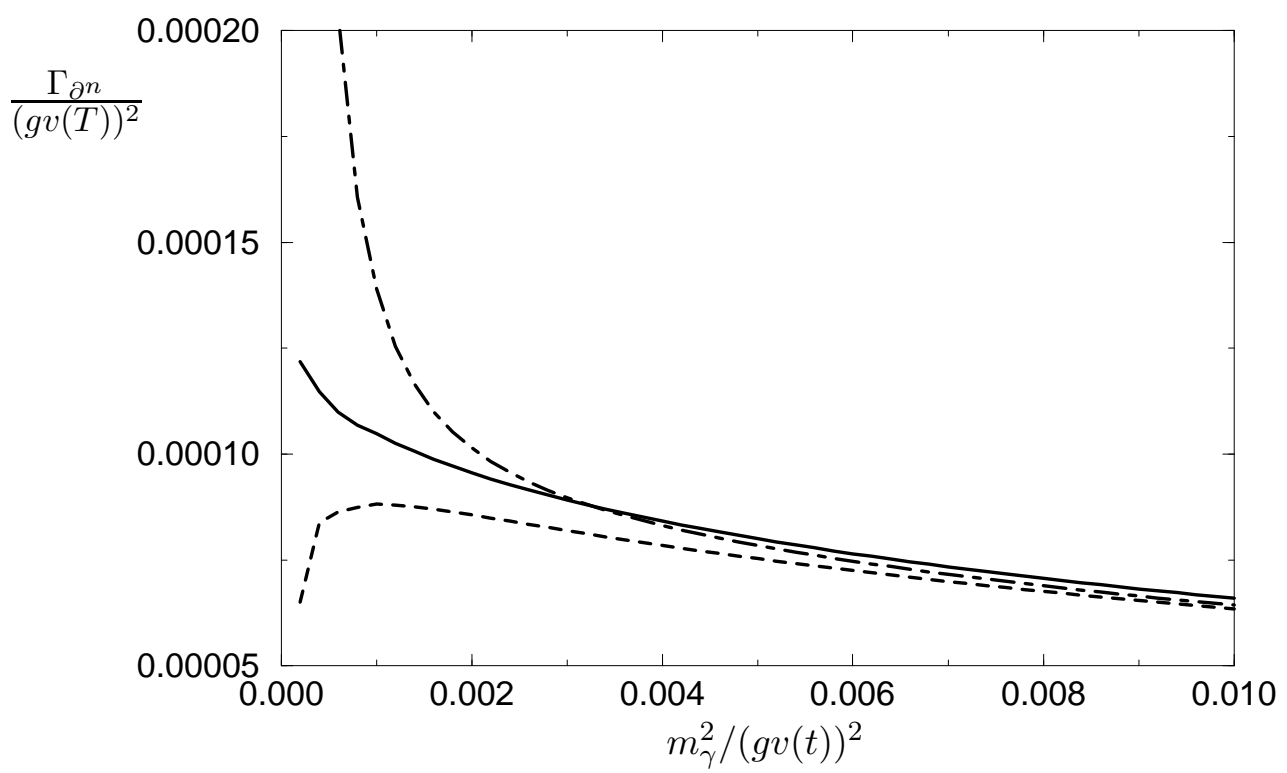

Figure 4: The one-loop contribution to the surface tension in the derivative expansion. The full line is the $Z$-factor contribution; the dashed line includes the terms up to order $\partial^{4}$; the dot-dashed line up to $\partial^{6}$.

The inequalities $(a)$ and $(b)$ in (47) have also been checked numerically and turn out to be quite accurate without any further factors.

In the multi local expansion $1 /(m d)$ has not to be small since all powers of the derivatives are summed but one still needs an expansion in powers of $\left(\varphi^{2}-m_{0}^{2}\right) / m^{2}$. Since we already know that higher derivative terms are small and that the $\partial \varphi \partial \varphi$ term dominates we can see very explicitly that this series converges very slowly for the massless mode.

Having introduced a cutoff mass the derivative expansion can be studied term by term because now the $z$-integrals no longer diverge. In fig. 1 we display the $Z$-factor contribution and the results including the next two orders of the derivative expansion for $\lambda_{T} / g^{2}=0.04$ and $d=12.5$ vs. $m_{\gamma}$. We find that a well-behaved converging expansion is obtained for $m_{\gamma}^{2} \gtrsim 0.002(g v)^{2}$. This is in agreement with the inequality obtained from 447.a). It also implies a cutoff for $\varphi<0.09$. This is a rather small cutoff, small compared to the dynamic mass scale set by the bound state masses in the symmetric phase.

The derivative expansion including the $Z$-factor is therefore a good approximation to the effective action if a small infrared mass $m_{\gamma}$ is introduced. The higher derivative terms are under complete control. Of course such a $m_{\gamma}$ influences the $Z$-factor contribution to the interface tension as well and thus parameterizes the effects outside the range of perturbation theory.

We restrict ourselves to the one-loop $Z$-factor in the rest of this paper, though considering its strong gauge fixing dependence a two-loop calculation would be highly desirable [26].

\section{The Interface Tension}

Taking into account the $Z$-factor modifies on the one hand the critical bubble or the planar interface solutions and gives on the other hand direct additional contributions to the effective action of the bubble surface or the interface. For definiteness we concentrate on the planar interface. Thin walled critical bubbles can be treated in the same way. 
Starting from the effective action (eq. (2)) neglecting the $\mathcal{O}\left(\partial^{4}\right)$-terms the saddle point equation reads

$$
Z_{\mathrm{H}}(\varphi) \partial^{2} \varphi+\frac{1}{2} \frac{\partial Z_{\mathrm{H}}(\varphi)}{\partial \varphi}\left(\partial_{i} \varphi\right)^{2}=\frac{\partial V_{\mathrm{eff}}(\varphi)}{\partial \varphi}
$$

With the substitution

$$
V_{\mathrm{eff}}(\varphi)=\tilde{V}(\varphi) Z_{\mathrm{H}}(\varphi)
$$

we obtain

$$
Z_{\mathrm{H}}(\varphi) \partial^{2} \varphi=Z_{\mathrm{H}}(\varphi) \frac{\partial \tilde{V}(\varphi)}{\partial \varphi}+\frac{\partial Z_{\mathrm{H}}(\varphi)}{\partial \varphi}\left(\tilde{V}(\varphi)-\frac{1}{2}\left(\partial_{i} \varphi\right)^{2}\right)
$$

The boundary conditions of a planar interface $\varphi(\vec{r})=\varphi(z)$ at the critical temperature are

$$
\varphi(z \rightarrow-\infty)=\varphi_{S}=0, \quad \varphi(z \rightarrow \infty)=\varphi_{B}=1
$$

where $\varphi_{S}$ and $\varphi_{B}$ are the field values of the symmetric and the broken minimum, respectively, and $V_{\text {eff }}\left(\varphi_{S}\right)=V_{\text {eff }}\left(\varphi_{B}\right)=0$. Eq. (52) is solved for a general positive $Z_{\mathrm{H}}(\varphi)$ by

$$
\frac{d \varphi}{d z}=\sqrt{2 \tilde{V}(\varphi(z))}
$$

The interface tension of this solution is

$$
\sigma=\int_{-\infty}^{\infty} d z\left(\frac{1}{2} Z_{\mathrm{H}}\left(\partial_{z} \varphi(z)\right)^{2}+V_{\mathrm{eff}}(\varphi(z))\right)
$$

where $V_{\text {eff }}(\varphi)$ has to be evaluated at the critical temperature. Substituting the integration variable one gets an expression which does not refer to the explicit interface solution [20]

$$
\sigma=\int_{\varphi_{S}}^{\varphi_{B}} d \varphi \sqrt{2 Z_{\mathrm{H}}(\varphi) V_{\mathrm{eff}}(\varphi)}
$$

$\sigma$ of eq. (56) is the generalization of the $Z=1$ formula used in the literature as perturbative value of the interface tension

$$
\tilde{\sigma}=\int_{\varphi_{S}}^{\varphi_{B}} d \varphi \sqrt{2 V_{\mathrm{eff}}(\varphi)}
$$

Eq. (56) and (57) are now used to investigate the influence of the one-loop corrections to the kinetic term on the interface tension. Using the two-loop effective potential of ref. 19] and the one-loop $Z$-factor of eq. (5) we calculated $\sigma$ (lower two lines) and $\tilde{\sigma}$ (upper two lines) for several values of $\lambda_{T}$ and the gauge fixing parameters $\xi=0$ (Landau gauge, full lines) and $\xi=1$ (Feynman gauge, dashed lines). In the latter case the real part has been taken where $Z$ becomes negative. The results are shown in fig. 5 .

In both gauges the interface tension is lowered due to the $Z$-factor contribution. The dependence on the gauge fixing parameter via the effective potential is over-compensated. It will be interesting to see whether this behavior improves if the two-loop $Z$-factor is used. The two data points in fig. 5 are the lattice values of the interface tension calculated in ref. [4]. The data point of ref. [9] was calculated on a four dimensional lattice and is difficult to compare. It is at a Higgs mass of $35 \mathrm{GeV}$ and roughly agree with the two-loop perturbative contribution. At large Higgs mass the lattice data fall significantly below the perturbative result. The $60 \mathrm{GeV}$ data point of ref. [4] is shown in fig. 5 . The trend towards a smaller interface tension is even more pronounced at larger Higgs masses [27]. One should keep in mind that these lattice data are plagued with systematical uncertainties 


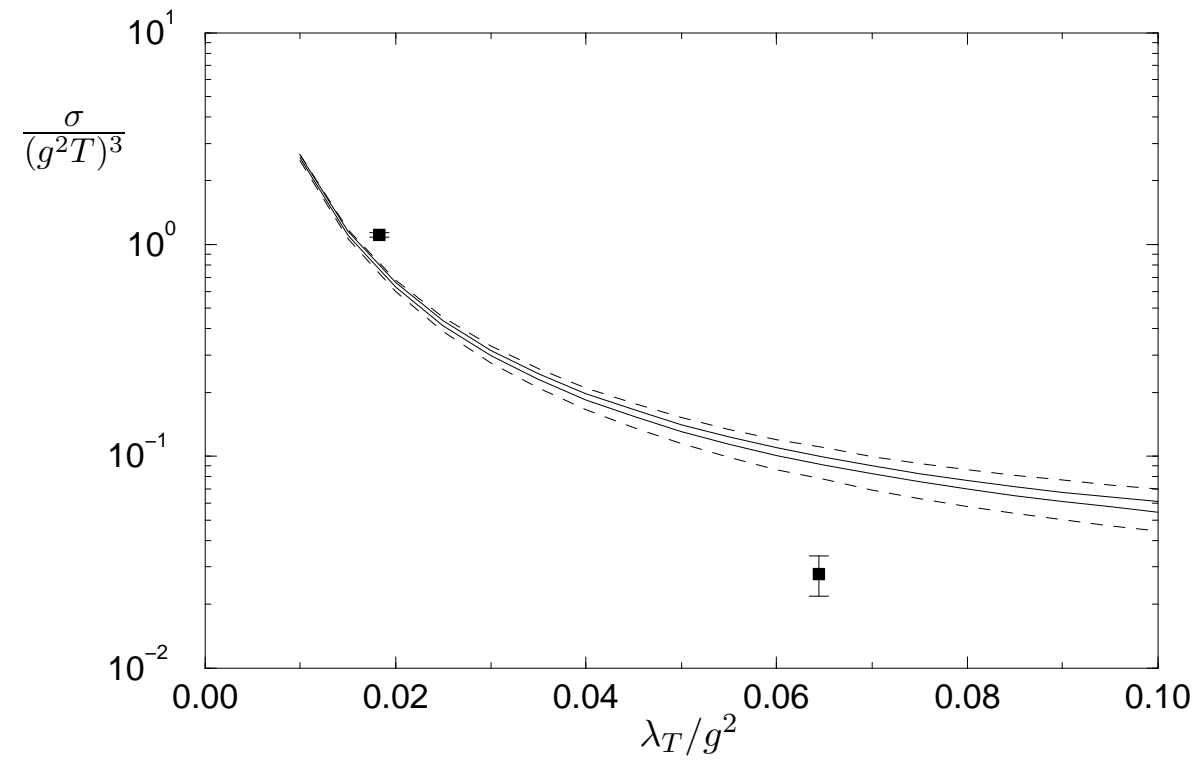

Figure 5: The interface tension vs. $\lambda_{T} / g^{2} . \quad \sigma$ of eq. (56) is plotted in the upper two lines; $\tilde{\sigma}$ of eq. (57) in the lower two lines. The dashed lines are the Feynman gauge results, the full lines are the Landau gauge values. The two data points are the lattice values of ref. 溲.

more important than the statistical error. Nevertheless, these results indicate that a quasiclassical effective potential approach may become completely unreliable in a Higgs mass range starting at about $60 \mathrm{GeV}$. A magnetic mass of the order $m_{\gamma}^{2} \sim 0.002(g v)^{2}$ (cf. fig. (4) would not be sufficient to explain such effects. In view of the good agreement of other quantities, e.g. the latent heat, this may appear surprising. One should keep in mind, however, that the interface tension is the only quantity out of the measured ones really testing the shape of the effective potential.

\section{Conclusion}

We started with the observation that the $Z$-factor is very gauge dependent and rather infrared sensitive. The derivative expansion for integrated quantities like the interface tension breaks down at the $\mathcal{O}\left(\partial^{4}\right)$ order due to massless modes obtained if one continues the perturbative description to small $\varphi$-values. One can treat the technical problem how to resum the higher derivative terms and we argued that there is a finite contribution of the massless channel to the interface tension.

Our numerical evaluations strongly indicate that this contribution to the higher derivative terms is small. In praxi the order of the multi local and the heat kernel expansion is limited and this kept us from proving this with our methods. A large contribution, if it arises nevertheless, would be a pure infrared effect. It would be based on a $\varphi$-range which is perturbatively not accessible and hence be an artifact which had to be substituted by a nonperturbative calculation in the hot phase. Introducing a infrared cut-off mass one suppresses such a contribution.

For small zero-temperature Higgs-masses $(\lesssim 70 \mathrm{GeV})$ perturbation theory works well in the broken phase. The infrared problems which plague the higher order derivative terms are restricted to even smaller $\varphi$-values $(<0.1 g v(T))$. The massive modes are free of infrared divergences in the whole $\varphi$-range. Our numerical calculations showed that the higher derivative terms give only very small contributions to the interface tension for these 
modes. They add a maximum of some percent in any order we calculated to the leading $Z$-factor contribution. In derivative expansion they are suppressed by $1 / d^{2}$ and our results confirmed that they indeed stay at this order of magnitude. The extrapolated results are in good agreement with the $Z$-factor predictions.

We conclude that the relevant perturbative contributions to the interface tension are not based on higher derivative terms but are the effective potential and the $\partial \varphi \partial \varphi$ term as usually is assumed in the literature.

The $Z$-factor in front of the latter term has to be taken into account properly. This can be done analytically using a semi-classical solution. The values of the interface tension is lowered by this effect but reliable calculations are not possible yet due to the strong gauge fixing dependence of the one-loop $Z$-factor. A two-loop calculation of this quantity may be helpful. Our results show that including the corrections to the $\partial \varphi \partial \varphi$ term is not sufficient to reach agreement with the published lattice interface tension at intermediate Higgs masses.

In view of a lot of unexpected agreement between lattice data and perturbative results and also some uncertainties in particular in the case of the interface tension it would be desirable to have a formalism bridging between perturbative treatment in the Higgs phase and strong interaction dynamics in the hot phase.

\section{Acknowledgments}

We would like to thank Mikko Laine for useful discussions.

\section{References}

[1] G.W. Anderson and L.J. Hall, Phys.Rev. D45 (1992) 2685.

M. Dine, R.G. Leigh, P. Huet, A. Linde and D. Linde, Phys.Rev. D46 (1992) 550.

M.E. Carrington and J.I. Kapusta, Phys.Rev. D47 (1993) 5304.

[2] K. Farakos, K. Kajantie, K. Rummukainen and M. Shaposhnikov, Nucl.Phys. B 425 (1994) 67.

[3] K. Kajantie, M. Laine, K. Rummukainen and M. Shaposhnikov, CERN-TH/95-226, hep-ph/9508379.

[4] K. Kajantie, M. Laine, K. Rummukainen and M. Shaposhnikov, CERN-TH/95-263, hep-lat/9510020.

[5] W. Buchmüller and O. Philipsen, Nucl.Phys. B 443 (1995) 47.

[6] W. Buchmüller, Z. Fodor and A. Hebecker, DESY-95-028, hep-ph/9502321;

Phys.Lett. B 331 (1994) 131.

[7] Z. Fodor, J. Hein, K. Jansen, A. Jaster, I. Montvay and F. Csikor, Phys.Lett. B 334 (1994) 405.

[8] Z. Fodor, J. Hein, K. Jansen, A. Jaster and I. Montvay Nucl.Phys. B 439 (1995) 147.

[9] F. Csikor, Z. Fodor, J. Hein and J. Heitger, Phys.Lett. B 357 (1995) 156

[10] E.-M. Ilgenfritz, J. Kripfganz, H. Perlt and A. Schiller Phys.Lett. B 356 (1995) 561

[11] B. Bergerhoff and C. Wetterich, HD-THEP-95-37, hep-ph/9508352 
[12] M. Joyce, T. Prokopec, and N. Turok, Phys.Lett. B 339 (1994) 312.

[13] G. Moore and T. Prokopec Phys.Rev.Lett. 75 (1995) 777.

[14] K. Enqvist and I. Vilja, Phys.Lett. B 344 (1995) 98.

[15] H.G. Dosch, J. Kripfganz, A. Laser and M.G. Schmidt, hep-ph/9509352, to appear in Phys.Lett..

[16] P. Arnold and O. Espinosa, Phys.Rev. D 47 (1993) 3546.

[17] Z. Fodor and A. Hebecker, Nucl.Phys. B 432 (1994) 127.

[18] M. Laine, Phys.Lett. B 335 (1994) 173; Phys.Rev. D 51 (1995) 4525.

[19] J. Kripfganz, A. Laser and M.G. Schmidt, Phys.Lett. B 351 (1995) 266.

[20] J. Kripfganz, A. Laser and M.G. Schmidt, Nucl.Phys. B 433 (1995) 467.

[21] A.O. Barvinsky and G.A. Vilkovisky, Nucl.Phys. B 282 (1987) 163; Nucl.Phys. B 330 (1990) 471, 512.

[22] R. Seeley, Am.Math.Soc.Proc. Pure Math. 10 (1967) 288.

B. De Witt, Dynamical Theory of Groups and Fields (Gordon and Breach, New York, 1965)

[23] D. Fliegner, P. Haberl, M.G. Schmidt and C. Schubert, DESY-94-221, hepth/9411177.

[24] D. Fliegner, M.G. Schmidt and C. Schubert, Z.Phys. C 64 (1994) 111.

[25] W. Buchmüller, Z. Fodor, T. Helbig and D. Walliser, Ann.Phys. 234 (1994) 260.

[26] A. Laser and M.G. Schmidt, in progress.

[27] M. Gürtler, E.-M. Ilgenfritz, J. Kripfganz, H. Perlt and A. Schiller Proceedings Buckow (1995) 